\title{
Statistical 2D and 3D Shape Analysis Using Non-Euclidean Metrics
}

\author{
Rasmus Larsen, Klaus Baggesen Hilger, and Mark C. Wrobel \\ Informatics and Mathematical Modelling, Technical University of Denmark \\ Richard Petersens Plads, Building 321, DK-2800 Kgs. Lyngby, Denmark \\ $\{\mathrm{rl}, \mathrm{kbh}, \mathrm{mcw}\} @$ @imm.dtu.dk, http://www . imm.dtu.dk
}

\begin{abstract}
The contribution of this paper is the adaptation of data driven methods for non-Euclidean metric decomposition of tangent space shape coordinates. This basic idea is to take extend principal components analysis to take into account the noise variance at different landmarks and at different shapes. We show examples where these non-Euclidean metric methods allow for easier interpretation by decomposition into biologically meaningful modes of variation. The extensions to PCA are based on adaptation of maximum autocorrelation factors and the minimum noise fraction transform to shape decomposition. A common basis of the methods applied is the assessment of the annotation noise variance at individual landmarks. These assessments are based on local models or repeated annotations by independent operators.
\end{abstract}

\section{Introduction}

For the analysis and interpretation of multivariate observations a standard methods has been the application of principal component analysis (PCA) to extract latent variables. Cootes et al. applied PCA to the analysis of tangent space shape coordinates [1. For various purposes different procedures for PCA using non-Euclidean metrics have been proposed. The maximum autocorrelation factor (MAF) transform proposed by Switzer [2] defines maximum spatial autocorrelation as the optimality criterion for extracting linear combinations of multispectral images. Contrary to this PCA seeks linear combinations that exhibit maximum variance. Because imaged phenomena often exhibit some sort of spatial coherence spatial autocorrelation is often a better optimality criterion than variance. We have previously adapted the MAF transform for analysis of tangent space shape coordinates 3]. In [4] the noise adjusted PCA or the minimum noise fraction (MNF) transformations were used for decomposition of multispectral satellite images. The MNF transform is a PCA in a metric space defined by a noise covariance matrix estimated from the data. For image data the noise process covariance is conveniently estimated using spatial filtering. In [5] the MNF transform is applied to texture modelling in active appearance models [6]. Bookstein proposed using bending energy and inverse bending energy as metrics in the tangent space [7]. Using the bending energy puts emphasis on the large scale variation, using inverse bending energy puts emphasis of small scale variation. 


\section{Methods}

\subsection{Minimum Autocorrelation Factors}

Let the spatial covariance function of a multivariate stochastic variable, $\boldsymbol{Z}_{k}$, where $k$ denotes spatial position and $\Delta$ a spatial shift, be $\Pi(\Delta)=\operatorname{Cov}\left\{\boldsymbol{Z}_{k}\right.$, $\boldsymbol{Z}_{k+\Delta}$ \}. Then by letting the covariance matrix of $Z_{k}$ be $\boldsymbol{\Sigma}$ and defining the covariance matrix $\boldsymbol{\Sigma}_{\Delta}=D\left\{\boldsymbol{Z}_{k}-\boldsymbol{Z}_{k+\Delta}\right\}$, we find

$$
\boldsymbol{\Sigma}_{\Delta}=2 \boldsymbol{\Sigma}-\boldsymbol{\Pi}(\Delta)-\boldsymbol{\Pi}(-\Delta)
$$

Then the autocorrelation in shift $\Delta$ of a linear combination of $Z_{k}$ is

$$
\operatorname{Corr}\left\{\boldsymbol{w}_{i}^{T} \boldsymbol{Z}_{k}, \boldsymbol{w}_{i}^{T} \boldsymbol{Z}_{k+\Delta}\right\}=1-\frac{1}{2} \frac{\boldsymbol{w}_{i}^{T} \boldsymbol{\Sigma}_{\Delta} \boldsymbol{w}_{i}}{\boldsymbol{w}_{i}^{T} \boldsymbol{\Sigma} \boldsymbol{w}_{i}}
$$

The MAF transform is given by the set of conjugate eigenvectors of $\boldsymbol{\Sigma}_{\Delta}$ wrt. $\boldsymbol{\Sigma}$, $W=\left[\boldsymbol{w}_{1}, \ldots, \boldsymbol{w}_{m}\right]$, corresponding to the eigenvalues $\kappa_{1} \leq \cdots \leq \kappa_{m}[2]$. The resulting new variables are ordered so that the first MAF is the linear combination that exhibits maximum autocorrelation. The $i$ th MAF is the linear combination that exhibits the highest autocorrelation subject to it being uncorrelated to the previous MAFs. The autocorrelation of the $i$ th component is $1-\frac{1}{2} \kappa_{i}$.

\subsection{Minimum Noise Fractions}

As before we consider a multivariate stochastic variable, $\boldsymbol{Z}_{k}$. We assume an additive noise structure $\boldsymbol{Z}_{k}=\boldsymbol{S}_{k}+\boldsymbol{N}_{k}$, where $\boldsymbol{S}_{k}$ and $\boldsymbol{N}_{k}$ are uncorrelated signal and noise components, with covariance matrices $\boldsymbol{\Sigma}_{S}$ and $\boldsymbol{\Sigma}_{N}$, respectively. Thus $\operatorname{Cov}\left\{\boldsymbol{Z}_{k}\right\}=\boldsymbol{\Sigma}=\boldsymbol{\Sigma}_{S}+\boldsymbol{\Sigma}_{N}$. By defining the signal-to-noise ratio (SNR) as the ratio of the signal variance and the noise variance we find for a linear combination of $\boldsymbol{Z}_{k}$

$$
\mathrm{SNR}_{i}=\frac{V\left\{\boldsymbol{w}_{i}^{T} \boldsymbol{S}_{k}\right\}}{V\left\{\boldsymbol{w}_{i}^{T} \boldsymbol{N}_{k}\right\}}=\frac{\boldsymbol{w}_{i}^{T} \boldsymbol{\Sigma}_{S} \boldsymbol{w}_{i}}{\boldsymbol{w}_{i}^{T} \boldsymbol{\Sigma}_{N} \boldsymbol{w}_{i}}=\frac{\boldsymbol{w}_{i}^{T} \boldsymbol{\Sigma} \boldsymbol{w}_{i}}{\boldsymbol{w}_{i}^{T} \boldsymbol{\Sigma}_{N} \boldsymbol{w}_{i}}-1
$$

So the minimum noise fractions are given by the set of conjugate eigenvectors of $\boldsymbol{\Sigma}$ wrt. $\boldsymbol{\Sigma}_{N}, W=\left[\boldsymbol{w}_{1}, \ldots, \boldsymbol{w}_{m}\right]$, corresponding to the eigenvalues $\kappa_{1} \geq \cdots \geq$ $\kappa_{m}$ [4. The resulting new variables are ordered so that the first MNF is the linear combination that exhibits maximum SNR. The $i$ th MNF is the linear combination that exhibits the highest SNR subject to it being uncorrelated to the previous MNFs. The SNR of the $i$ th component is $\kappa_{i}-1$.

The central problem in the calculation of the MNF transformation is the estimation of the noise with the purpose of generating a covariance matrix that approximates $\boldsymbol{\Sigma}_{N}$. Usually the spatial nature of the data is utilized and the noise is approximated by the difference between the original measurement and a spatially filtered version or a local parametric function (e.g. plane, quadratic function). 


\subsection{MNF and MAF for Shape Decomposition}

We have previously [3] shown how to adapt MAF to shape decomposition by utilizing the ordering of landmarks (variables) instead of ordering of pixels (observations) by transposing the data matrix. Furthermore, it was shown that Molgedey-Schuster's [8] independent components (ICA) is equivalent to MAF. If the matrices in Equations (2) and (3) are singular the solution must be found in the affine support of the matrix in the denominator, e.g. by means of a generalized singular value decomposition.

\section{Materials}

We demonstrate the properties of the techniques that we propose on two datasets. The first dataset consists of $2 \mathrm{D}$ annotations of the outline of the right and left lung from 115 standard PA chest radiographs. The chest radiographs were randomly selected from a tuberculosis screening program and contained normal as well as abnormal cases. The annotation process was conducted by identification of three anatomical landmarks on each lung outline followed by equidistant distribution of pseudo landmarks along the 3 resulting segments of the outline. In Fig. 1(b) the landmarks used for annotation are shown. Each lung field is annotated independently by two observers - Dr. Bram van Ginneken and Dr. Bart M. ter Haar Romeny. The dataset was supplied to us by Dr. Bram van Ginneken. For further information the reader is refered to the Ph.D. thesis of van Ginneken [9].

The second dataset consist of 4D landmarks of a set of surfaces of human mandibles (the lower jaw) registered over time. The surfaces were extracted in a previous study by Dr. Per R. Andresen from CT scans of 7 Apert patients imaged from 3-5 times from age 3 months to 12 years. The mandibles are assumed to exhibit normal growth. The scans were performed for diagnostic and treament planning purposes and supplied by Dr. Sven Kreiborg (School of Dentistry, University of Copenhagen, Denmark) and Dr. Jeffrey L. Marsh (Plastic and Reconstructive Department for Pediatric Plastic Surgery, Washington University School of Medicine at St. Louis Children's Hospital, St. Louis, Missouri, USA). The surface extraction and registration was carried out using matching of the extremal mesh followed by a geometry-constrained diffusion procedure described in [10 11]. The surfaces contain approximately 14.000 homologous points.

\section{Results}

\subsection{Lung Dataset}

We intend to use the annotation by two independent observers to estimate the annotation uncertainty. Initially the lung annotations are aligned to a common reference frame by concatenating the annotations of the two observers and performing a generalized Procrustes analysis (GPA) [12,13]. Now we can compute 


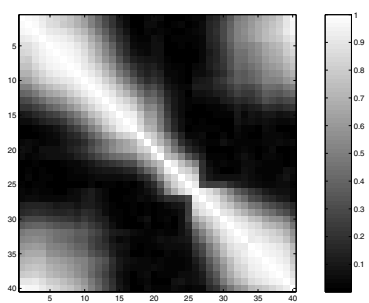

(a)

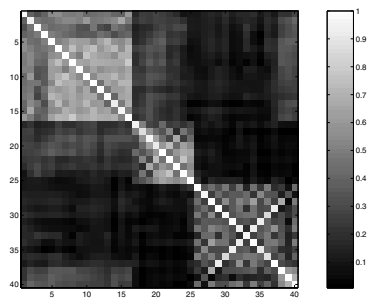

(d)

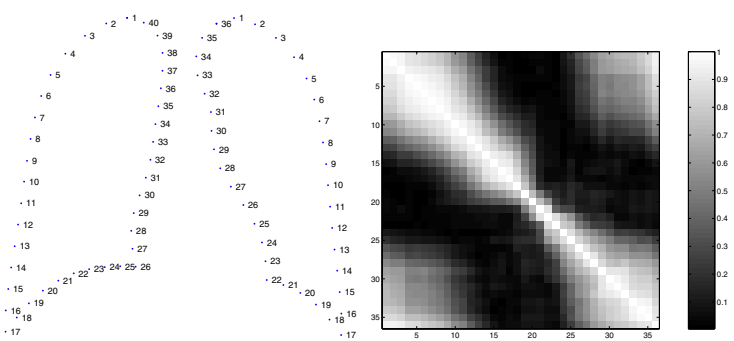

(b)

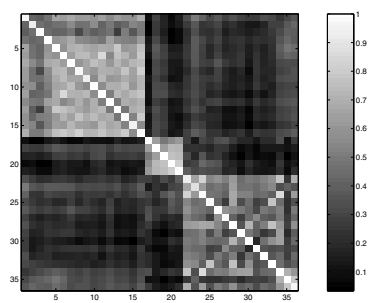

(e)

Fig. 1. Landmarks of the left and right lung. Landmark numbers are shown in the middle. The right lung is annotated by 40 landmarks, and the left lung by 36 . The anatomical landmarks on the right field are points 1,17 , and 26 , on the left field the anatomical landmarks are points 1, 17, and 22. (a),(c) Inter-observer difference canonical correlations between landmarks for the right and left lungs. (d),(e) Interneighbour landmark difference canonical correlations between landmark for the right and left lung.

the differences between the two sets of annotations and estimate an inter-observer covariance matrix of the landmark coordinates. Obviously we would like to view the intercorrelation per landmark and not per coordinate. Rotation of the frame of reference will shift the correlation between $x$ and $y$ coordinates which may cause some confusion. In order to overcome this problem for each pair of landmarks we estimate the maximum correlation between linear combinations of their coordinates. These are the canonical correlations 14.

In Fig. 1 we see these correlations for the right and left lung. The inter lung correlations are neglible. For both set of lungs we see a high degree of correlation along the curved top outline of the lungs. For both lungs landmark 1 is the top point. Again for both lungs there is no or little correlation across the two anatomical landmarks that delimit the bottom segment of the outlines.

The inter-observer covariance matrix defines one sensible metric to use when decomposing the shape variability. This would put less emphasis of landmarks with high annotation variance and more emphasis on landmarks with low annotation variance, and result in a minimum noise fraction transform. As an alternative to assessing the interobserver differences we may consider the covariance of the difference of neighbouring landmarks. The correlation structure of these are also shown in Fig. 1, Here the partitioning of landmarks in three segments 


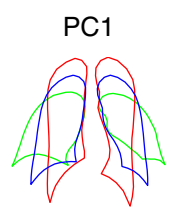

PCC1

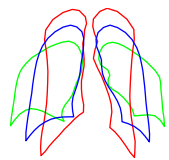

EPC1

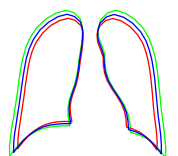

MAF1

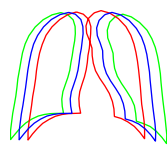

REL1

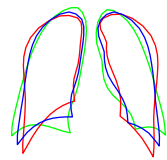

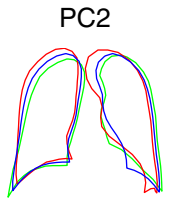

PCC2

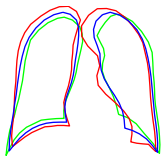

EPC2

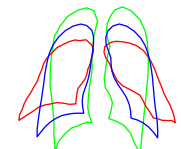

MAF2

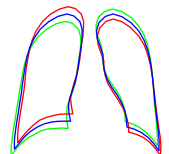

REL2

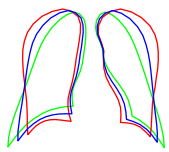

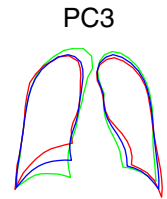

PCC3

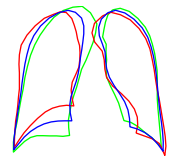

EPC3

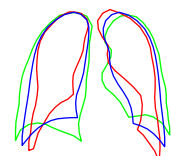

MAF3

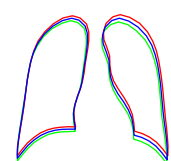

REL3

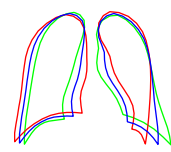

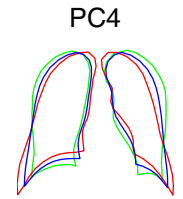

PCC4

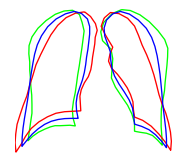

EPC4

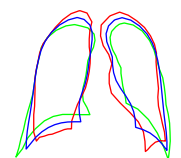

MAF4

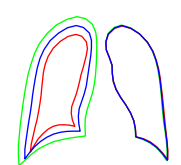

REL4

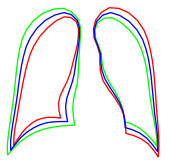

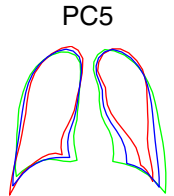

PCC5

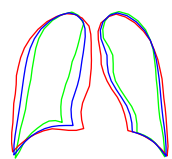

EPC5

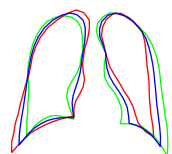

MAF5

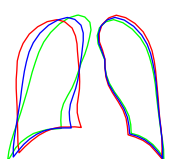

REL5

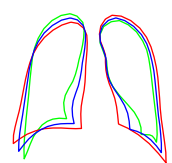

PC6

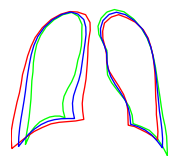

PCC6

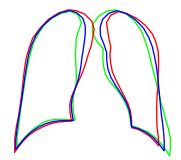

EPC6

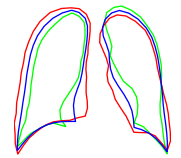

MAF6

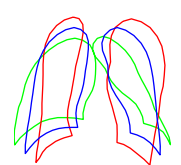

REL6

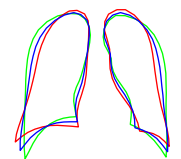

Fig. 2. The 6 most important principal components (PC), principal components on a standardized dataset (PCC), annotation noise adjusted principal components (EPC), maximum autocorrelation factors (MAF), and relative warps (REL). The blue curve is the mean shape, and the green and red curves represent \pm 5 standard deviations as observed in the training set.

for each lung is more pronounced. Using this covariance as metric corresponds to the MAF transform.

In Fig. 2 the 6 most important principal components (PC), principal components on a standardized dataset (PCC), annotation noise adjusted principal components (EPC), maximum autocorrelation factors (MAF), and relative warps (REL) are shown. The relative warps use the bending matrix of the estimated mean shape as metric. The PCs and PCCs are fairly similar, but the EPCs, MAFs, and RELs are different. The latter three all represent uses of metrics that are significantly different from the Euclidean one. The first EPC is a an aspect ratio variation, and the following $5 \mathrm{EPC}$ 's seeems to be a mix of the first PCs. The first MAF is also an aspect ratio variation, and the following MAF's also have evident large scale interpretataions. In particular, MAF4 is the relative size of the lungs. The relative warps also give various large scale variations but they are not as easily interpretable as the MAFs. 


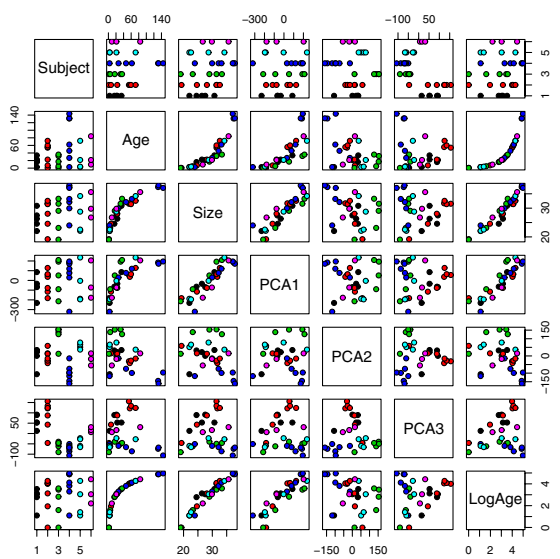

(a)

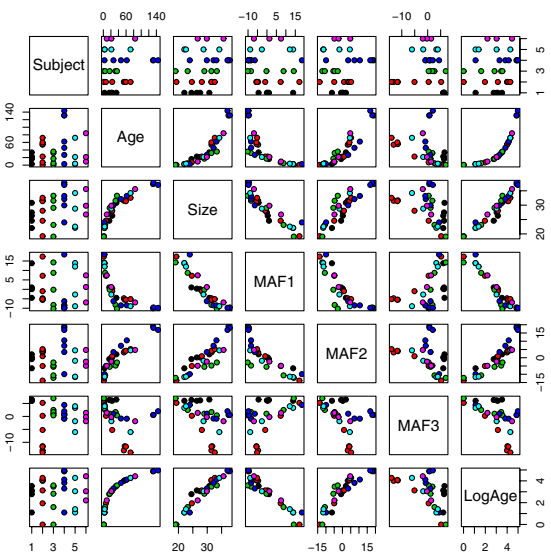

(b)

Fig. 3. Scatter plots of the 3 first PCs and age, log age, and centroid size. A strong correlation between the shape variation in PC1 and MNF1 to size is demonstrated. Because size has been filtered out of the shape decomposition in the Procrustes analysis these components can be interpreted as shape change due to growth. The lower order components exhibit variation between individuals.

\subsection{Mandible Dataset}

A major objective for the analysis/decomposition of the mandible dataset is the construction of a growth model that allows prediction of mandible size and shape from early scans (1-3 months). When performing pediatric cranio-facial surgery prediction of growth patterns is extremely important. Growth modelling will also add to basic understanding as well as have teaching implications. Here we will demonstrate the use of the MNF transformation for decomposition of a $3 \mathrm{D}$ dataset as an alternative to PCA.

The mandibles are aligned using a generalized 3D Procrustes analysis [15] and projected into tangent space. Each mandible is represented by a triangulated surface based on the 14000 landmarks. This triangulation allows us to determine the neighboring landmarks easily. We estimate the noise covariance matrix in Equation (3) as the covariance matrix of the deviations from the mean displacements between landmark coordinates and planes fitted locally to all landmarks in a neighbourhood. In the example shown we have used a 4th order neighbourhood. In Fig. 3 pairwise scatter plots of the first three components and age, log age, and centroid size are shown for PCs as well as MNFs. For the PCs we see that there is strong relationship between PC1, age and size. This means that PC1 relates to mandible growth, as was also concluded and utilized in [10]. PC2 and PC3 does not correlate to age or size but contain variation between individuals. For the MNFs we see that we have captured two uncorrelated modes of variation namely MNF1 and MNF2 that relate to size and age. MNF3 is a contrast between the three younger mandible scans of subject number 5 and the rest of the mandibles. In Figs. 4 and 5 the first two PCs and MNFs are shown. In each plot a 
greenish meanshape and a goldish positive or negative deviation are shown. For PC1 we see a contrast between young, broad, flat mandibles with small condyles and elder, slimmer, higher mandibles with large condyles and erupted teeth. For MNF1 and MNF2 we see different patterns of growth.

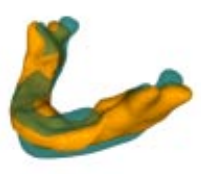

(a) PC1 '-'

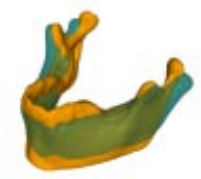

(b) $\mathrm{PC} 1$ '+'

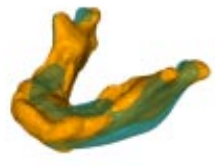

(c) PC2 '-'

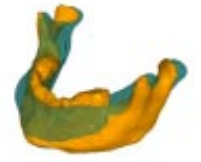

(d) $\mathrm{PC} 2$ ' + '

Fig. 4. Principal components 1 and 2 shown as \pm 2 standard deviations across the training set.

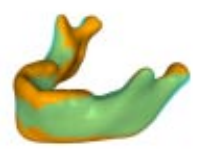

(a) MNF1 '-'

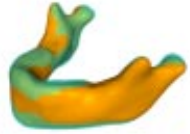

(b) MNF1 ' + '

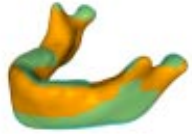

(c) MNF2 '-'

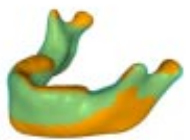

(d) MNF2 '+'

Fig. 5. Minimum noise fractions 1 and 2 shown as \pm 2 standard deviations across the training set.

\section{Conclusion}

We have demonstrated a series of data driven methods for constructing nonEuclidean metric linear decompositions of the tangent space shape variability in $2 \mathrm{D}$ and $3 \mathrm{D}$. We have demonstrated ways of constructing such a metric based on repeated measurements as well as by use of the spatial nature of the outline and surface models considered. It turns out that the MAF and MNF transforms are superior in terms of interpretability for decompoing large scale variation. These methods are tools for determining un-correlated biological modes of variation.

\section{Acknowledgements}

The work was supported by the Danish Technical Research Council under grant number 26-01-0198 which is hereby gratefully acknowledged. The authors thank Dr. Bram van Ginneken for use of the lung annotation data set. The authors also thank Dr. Sven Kreiborg and Tron Darvann (School of Dentistry, University of Copenhagen, Denmark) for providing insight into the study of mandibular growth. 


\section{References}

1. T. F. Cootes, G. J. Taylor, D. H. Cooper, and J. Graham, "Training models of shape from sets of examples," in British Machine Vision Conference: Selected Papers 1992, (Berlin), Springer-Verlag, 1992.

2. P. Switzer, "Min/max autocorrelation factors for multivariate spatial imagery," in Computer Science and Statistics (L. Billard, ed.), pp. 13-16, Elsevier Science Publishers B.V. (North Holland), 1985.

3. R. Larsen, H. Eiriksson, and M. B. Stegmann, "Q-MAF shape decomposition," in Medical Image Computing and Computer-Assisted Intervention - MICCAI 2001, 4th International Conference, Utrecht, The Netherlands, vol. 2208 of Lecture Notes in Computer Science, pp. 837-844, Springer, 2001.

4. A. A. Green, M. Berman, P. Switzer, and M. D. Craig, "A transformation for ordering multispectral data in terms of image quality with implications for noise removal," IEEE Transactions on Geoscience and Remote Sensing, vol. 26, pp. 6574, Jan. 1988.

5. K. B. Hilger, M. B. Stegmann, and R. Larsen, "A noise robust statistical texture model," in Medical Image Computing and Computer-Assisted Intervention - MICCAI 2002, 5th International Conference, Tokyo, Japan, 2002. 8 pp. (submitted).

6. T. F. Cootes, G. J. Edwards, and C. J. Taylor, "Active appearance models," in Proceedings of the European Conf. On Computer Vision, pp. 484-498, Springer, 1998.

7. F. L. Bookstein, Morphometric tools for landmark data. Cambridge University Press, 1991. 435 pp.

8. L. Molgedey and H. G. Schuster, "Separation of a mixture of independent signals using time delayed correlations," Physical Review Letters, vol. 72, no. 23, pp. 36343637, 1994.

9. B. van Ginneken, Computer-Aided Diagnosis in Chest Radiographs. PhD thesis, Image Sciences Institute, University Medical Center Utrecht, Utrecht University, Utrecht, the Netherlands, 2001. 184 pp.

10. P. R. Andresen, F. L. Bookstein, K. Conradsen, B. K. Ersbøll, J. L. Marsh, and S. Kreiborg, "Surface-bounded growth modeling applied to human mandibles," IEEE Transactions on Medical Imaging, vol. 19, Nov. 2000. 1053-1063.

11. P. R. Andresen and M. Nielsen, "Non-rigid registration by geometry-constrained diffusion," Medical Image Analysis, vol. 5, no. 2, pp. 81-88, 2001.

12. J. C. Gower, "Generalized Procrustes analysis," Psychometrika, vol. 40, pp. 33-50, 1975.

13. C. Goodall, "Procrustes methods in the statistical analysis of shape," Journal of the Royal Statistical Society, Series B, vol. 53, no. 2, pp. 285-339, 1991.

14. H. Hotelling, "Relations between two sets of variables," Biometrika, vol. 28, pp. 321-377, 1936.

15. J. M. F. T. Berge, "Orthogonal Procrustes rotation for two or more matrices," Psychometrika, vol. 42, pp. 267-276, 1977. 